journal club

\title{
Depressive Stimmungslage und Negativsymptome werden verbessert
}

Fragestellung: Untersuchung der Sicherheit und Wirksamkeit von Antidepressiva bei gleichzeitiger Behandlung mit Antipsychotika in der Indikation Schizophrenie.

Hintergrund: Bei an Schizophrenie erkrankten Personen liegt die Prävalenz depressiver Symptomatik bei $25 \%$. Bei etwa $30 \%$ der Patienten mit Schizophrenie werden Antidepressiva verschrieben. Jedoch gibt es bisher keine evidenzbasierten Empfehlungen zur Verordnung von Antidepressiva bei Schizophrenie. Es ist nicht ausreichend untersucht, welche Antidepressiva die wirkungsvollsten sind und welche spezifischen Effekte in Kombination mit einer antipsychotischen Behandlung zu erwarten sind. Aufgrund der Häufigkeit von Depressionen und Negativsymptomatik bei Patienten mit Schizophrenie, analysiert dieses Review alle randomisiert kontrollierten Studien, bei denen Antidepressiva als Begleitmedikation einer antipsychotischen Behandlung untersucht wurden.

Patienten und Methodik: In dem systematischen Review wurden die relevanten Datenbanken (Embase, MEDLINE, BIOSIS, PsycINFO, PubMed, Cochrane Central Register of Controlled Trials, WHO International Trial Registry, ClinicalTrials.govLibrary) nach Publikationen

Helfer B, Samara MT, Huhn M et al. Efficacy and safety of antidepressants added to antipsychotics for schizophrenia: A systematic review and meta-analysis. Am J Psychiatry 2016; 173: 876-86 durchsucht, die vor dem 24. Juni 2015 publiziert wurden. Einschlusskriterien waren Studien mit mindestens einer Interventionsdauer von einer Woche, die Patienten mit einer Diagnose aus dem Schi- zophreniespektrumbereich einbezogen. Es wurden ausschließlich randomisiert kontrollierte Studien selektiert, die eine Kombination aus Antipsychotikum und Antidepressivum mit einer Kombination aus Antipsychotikum und Placebobedingung oder mit einer antipsychotischen Monotherapie verglichen. Es wurden Subgruppenmetaanalysen und Sensitivitätsanalysen durchgeführt. Außerdem wurden der „Publication-Bias“ und der „Risk-of-Bias“ untersucht.

Ergebnisse: 82 randomisierte Studien mit insgesamt 3.608 eingebundenen Patienten. Add-on-Antidepressivabehandlungen waren wirksamer als die Kontrollbehandlungen hinsichtlich depressiver Symptome (95\%-Konfidenzintervall [KI] - 0,38 bis - 0,12), Negativsymptomatik ( $95 \%-\mathrm{KI}-0,44$ bis - 0,16), genereller Symptome (95\%-KI - 0,39 bis - 0,09) und positiver Symptome (95\%-KI - 0,33 bis - 0,01), der Lebensqualität (95\%-KI - 0,57 bis - 0,06) und der Ansprechrate (95\%-KI 1,29 bis 1,78). Es gab keine statistischen Unterschiede bezüglich der Re-Exazerbation einer Psychose, einer verfrühten Abbruchrate und der Patientenanzahl, die mindestens eine Nebenwirkung hatten. Mehr Patienten mit einer Add-on-Behandlung litten unter abdominalen Schmerzen, Obstipation, Schwindel und Mundtrockenheit.

Schlussfolgerungen: Eine zusätzliche Medikation mit Antidepressiva bei einer antipsychotischen Behandlung ist wirksam hinsichtlich depressiver und Negativsymptomatik bei Patienten mit Schizophrenie, auch wenn die Effektgrößen klein waren. Die Effektgrößen waren höher, wenn nur Patienten mit einer deutlichen depressiven Symptomatik oder vorherrschenden $\mathrm{Ne}$ gativsymptomatik eingeschlossen wurden.

\section{- Kommentar von Joachim Cordes und Enoh Meuthen, Düsseldorf}

\section{Antidepressiva bei Antipsychotikatherapie differenzierter betrachten}

Die umfangreiche und methodisch gute Metaanalyse spricht für die Wirksamkeit einer zusätzlichen antidepressiven Behandlung bei Vorliegen von depressiver Symptomatik oder Negativsymptomatik im Rahmen der Schizophrenie. Trotz der Schätzung, dass nur einer von neun Patienten eine Verbesserung zeigt, kann die Empfehlung als klinisch bedeutsam angesehen werden. Auf die Positivsymptomatik zeigte eine zusätzliche Behandlung mit Antidepressiva keinen negativen Effekt. Die Metaanalyse umfasst mit insgesamt 3.608 Patienten eine große Stichprobenzahl im Vergleich zu bisherigen Reviews. Außerdem adressiert diese Arbeit spezifische Subgruppen hinsichtlich Patientenmerkmalen und zusätzlicher Medikation. Da 82 randomisiert kontrollierte Studien eingeschlossen wurden, erreicht der abgeleitete Empfehlungsgrad eine Evidenzstufe I (hohes Evidenzniveau). Subgruppenanalysen sollten aufgrund der geringen Stichproben zurück- haltend interpretiert werden. Hinsichtlich der Verbesserung von Negativsymptomatik unter Antidepressiva bedarf es weiterer Klärung, ob es sich um eine spezifische Wirkung oder eine Folge der Besserung von depressiver Symptomatik handelt.

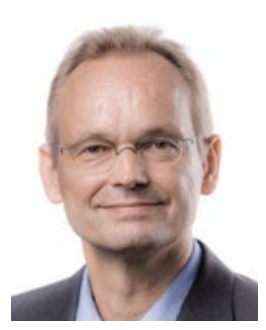

PD Dr. med. Joachim Cordes, Düsseldorf

Klinik und Poliklinik für Psychiatrie und

Psychotherapie, Heinrich Heine Universität, LVR-Klinikum Düsseldorf

E-Mail: joachim.cordes@lvr.de 